\title{
FCNC and EFT interpretations in CMS
}

\author{
Brieuc Francois*广 \\ Hanyang University \\ E-mail: brieuc.francoisehotmail.com
}

\begin{abstract}
Top quark production can probe physics beyond the Standard Model (SM) in different ways. Some processes, and especially certain angular correlations, are sensitive to the existence of anomalous top quark couplings. In the SM, flavor-changing neutral currents (FCNC) are forbidden at tree level and are strongly suppressed in loop corrections. Several extensions of the SM incorporate significantly enhanced FCNC behavior that can be directly probed in top quark processes. Current approaches adopting an EFT framework allow describing effects of new physics in a model independent way. This document reviews the current limits on FCNC searches in the top sector, and EFT interpretations.
\end{abstract}

XXVII International Workshop on Deep-Inelastic Scattering and Related Subjects - DIS2019 8-12 April, 2019

Torino, Italy

\footnotetext{
* Speaker.

${ }^{\dagger}$ On behalf of the CMS Collaboration.
} 


\section{Introduction}

Despite its numerous successes, the Standard Model (SM) is widely believed to be a low energy approximation of a more fundamental theory. The extensive searches for Beyond Standard Model (BSM) phenomena performed by the ATLAS and CMS [1] experiments suggest the absence of new particles at energies up to a few $\mathrm{TeV}$. This situation allows one to parametrize the effects of new physics at LHC energies by higher-dimension gauge-invariant operators built from SM fields. Neglecting the dimension-5 operator that violates lepton-number conservation, one can write the following general Effective Field Theory (EFT) Lagrangian:

$$
\mathscr{L}_{E F T}=\mathscr{L}_{S M}^{(4)}+\frac{1}{\Lambda^{2}} \sum_{k} c_{k}^{(6)} \mathscr{O}_{k}^{(6)}
$$

with $\Lambda$ the typical scale of new physics, $\mathscr{O}_{k}^{(6)}$ the dimension- 6 operators and $c_{k}^{(6)}$ their associated parameters that can be studied and constrained with the current CMS data.

\section{EFT constraints from CMS}

\section{1 $t \bar{t}$ and $t W$ as EFT probes}

The top-quark pair production $(t \bar{t})$ and the associated production of a top-quark with a $\mathrm{W}$ boson $(t W)$ processes can be used as probes for EFT due to their sensitivity to the following six CP even operators:

$$
\mathscr{O}_{\Phi q}^{(3)}, \mathscr{O}_{t W}, \mathscr{O}_{t G}, \mathscr{O}_{G}, \mathscr{O}_{u(c) G}
$$

CMS performed a search for new physics using these two processes in proton-proton collisions with two opposite-sign isolated leptons (electrons or muons) and b-jets. After this baseline selection, events are further categorized based on the number of (b-)jets and several Neural Networks (NN) are trained to separate $t W$ from $t \bar{t}, t W$ from the other backgrounds ( $t \bar{t}, W W$ and Drell-Yan) or the contribution of $\mathscr{O}_{u(c) G}$ from $t W+t \bar{t}$. The NN output distributions are used, for the first time directly from data, to extract best-fit values and confidence intervals on the effective couplings associated to Eq. 2.1, assuming one non-vanishing operator at a time, as shown on Tab. 1. More details on this analysis can be found in Ref. [2].

\begin{tabular}{llllll} 
Coupling & Obs. best fit & Obs. 68\% CI & Obs. 95\% CI & Exp. 68\% CI & Exp. 95\% CI \\
\hline$C_{G} / \Lambda^{2}$ & -0.18 & {$[-0.73,0.42]$} & {$[-1.01,0.70]$} & {$[-0.82,0.51]$} & {$[-1.07,0.76]$} \\
$C_{\Phi q}^{(3)} / \Lambda^{2}$ & -1.52 & {$[-2.71,-0.33]$} & {$[-3.82,0.63]$} & {$[-1.05,0.88]$} & {$[-2.04,1.63]$} \\
$C_{t W} / \Lambda^{2}$ & 2.38 & {$[0.22,4.57]$} & {$[-0.96,5.74]$} & {$[-1.14,5.93]$} & {$[-1.91,6.70]$} \\
$C_{t G} / \Lambda^{2}$ & -0.13 & {$[-0.27,0.02]$} & {$[-0.41,0.17]$} & {$[-0.15,0.14]$} & {$[-0.30,0.28]$} \\
$C_{u G} / \Lambda^{2}$ & -0.017 & {$[-0.13,0.13]$} & {$[-0.22,0.22]$} & {$[-0.21,0.21]$} & {$[-0.30,0.30]$} \\
$C_{c G} / \Lambda^{2}$ & -0.032 & {$[-0.26,0.26]$} & {$[-0.46,0.46]$} & {$[-0.46,0.46]$} & {$[-0.65,0.65]$}
\end{tabular}

Table 1: Observed and expected allowed intervals together with the observed best fit values for the effective couplings impacting $t W$ and $t \bar{t}$ processes [2]. 


\subsection{Chromo-magnetic dipole moment}

Several BSM such as Two Higgs Doublet Models, supersymmetry or technicolor predict anomalous top chromo-magnetic dipole moment (CMDM). In the EFT framework, anomalous top CMDM is introduced by the $\mathscr{O}_{t G}$. This operator modifies both the rate and kinematics of the $t \bar{t}$ process, especially via modification of its spin structure. As a consequence, spin-sensitive observable provide a very good proxy to study the top CMDM. An example of analysis exploiting this is the measurement of differential $t \bar{t}$ cross-sections in events with two opposite-sign leptons and (bjets [3]. The analysis measures, among others, the radial angular distance between the two leptons $(\Delta \Phi(l, \bar{l}))$ at particle-level in a fiducial phase space close to that of the detector acceptance. Figure 1 shows this distribution for data and theoretical predictions with various values of $C_{t G}$ together with the associated best-fit and confidence intervals on $C_{t G}$ derived from a $\chi^{2}$ minimization technique on that distribution. The predictions for $t \bar{t}$ production with anomalous CMDM are derived at NLO accuracy in QCD, featuring a stronger CMDM effect and reduced scale uncertainties compared to the LO predictions. Another analysis measured 22 spin-sensitive variables in events with two
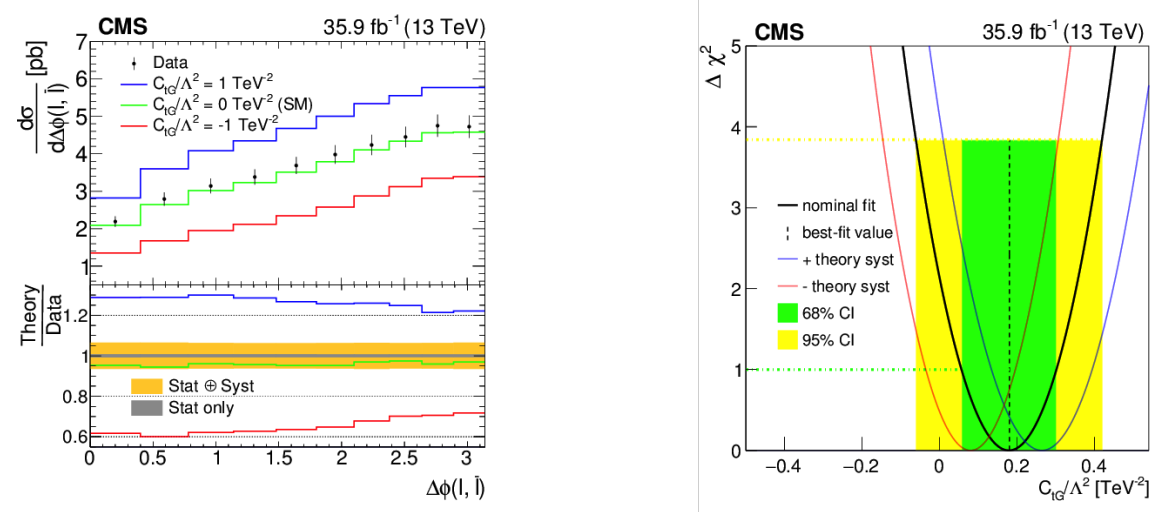

Figure 1: The left-hand side plot shows the differential $t \bar{t}$ cross sections as a function of $\Delta \Phi(l, \bar{l})$ at the particle level in the fiducial phase space. The right-hand side plot shows the $\Delta \chi^{2}$ values from the fit to the data in the left plot, as a function of $C_{t G}$ [3].

leptons and used 20 of them (removing laboratory-based observables) to constrain $C_{t G}$, porting the sensitivity to $-0.07<\frac{C_{G G}}{\Lambda^{2}}<0.16 \mathrm{TeV}^{-2}$ at $95 \%$ confidence level as shown on Fig 2. More details on this analysis can be found in Ref. [4].

\subsection{Constraining EFT with rare processes}

Rare processes can also play an important role in the study of top-related EFT operators as shown by the search for SM production of four top quarks ( $t \bar{t} t \bar{t})$ [5] performed in events with one or two leptons and jets. The analysis uses Boosted Decision Trees (BDT) to identify three-jets combination from hadronic top and to separate $t \bar{t} t \bar{t}$ from $t \bar{t}$. 95\% CL upper limit on $t \bar{t} t \bar{t}$ production cross-section are derived from the latter BDT distribution and is combined with the one from an orthogonal search in same-sign and multi-lepton final state [6]. The observed(expected) combined upper limit, 3.6(2.2) $\times \sigma_{t \bar{t} \bar{t}}^{\mathrm{SM}}$, is then used to constrain four EFT operators assuming one non-vanishing coupling at a time as shown on Tab. 2. To derive the cross section predictions in the 


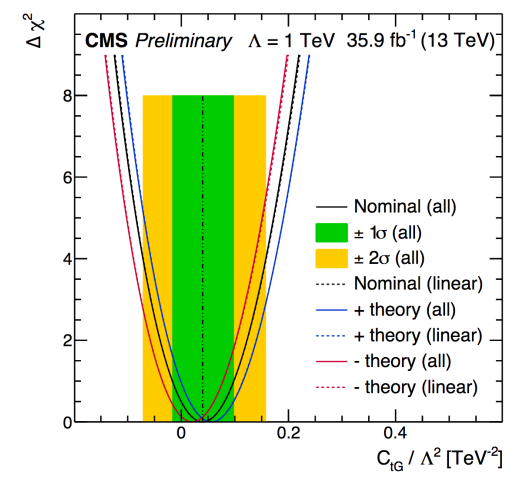

Figure 2: $\Delta \chi^{2}$ as a function of $C_{t G}$ obtained from the simultaneous fit of 20 spin-sensitive variables [4].

\begin{tabular}{ccc} 
Operator & Expected $\frac{C_{k}}{\Lambda^{2}}\left(\mathrm{TeV}^{-2}\right)$ & Observed $\frac{C_{k}}{\Lambda^{2}}\left(\mathrm{TeV}^{-2}\right)$ \\
\hline $\mathscr{O}_{t t}^{1}$ & {$[-1.5,1.3]$} & {$[-2.1,2.0]$} \\
$\mathscr{O}_{Q Q}^{1}$ & {$[-1.5,1.3]$} & {$[-2.2,2.0]$} \\
$\mathscr{O}_{Q t}^{1}$ & {$[-2.4,2.4]$} & {$[-2.1,2.0]$} \\
$\mathscr{O}_{Q t}^{8}$ & {$[-5.6,4.3]$} & {$[-7.9,6.6]$}
\end{tabular}

Table 2: Expected and observed 95\% CL intervals for selected coupling parameters. The intervals are extracted from upper limit on the $t \bar{t} t \bar{t}$ production cross section in the EFT model, where only one selected operator has a non-vanishing contribution [5].

EFT framework, the anomalous interactions are implemented in the FEYNRULES [7] package and interfaced to MG5_AMC@NLO [8]. Confidence levels have also been derived with the contribution of other operators marginalized, leading to very similar results. Another rare process that can be used to probe EFT operators is the production of $Z$ boson in association with a top quark pair $(t \bar{t} Z)$. This process has been studied with the 2016 and 2017 datasets $\left(77.5 \mathrm{fb}^{-1}\right)$ in the three and four leptons final states [9]. Fifteen control and signal regions are defined based on the lepton multiplicity, the (b-)jet content, the transverse momentum of the $Z$ boson and the angle between the negatively charged lepton and the $\mathrm{Z}$ candidate. A binned likelihood fit is performed on the yields in each of these regions and best-fit values together with confidence intervals are extracted on four dimension- 6 operator parameters inducing anomalous $t Z$ or $t \gamma$ interactions, assuming up to two non-vanishing operators at a time. Signal predictions are derived based on the reweighting of a SM NLO $t \bar{t} Z$ sample with weights obtained by comparing LO SM with LO EFT samples. Figure 3 shows the results in two two-dimensional planes, consistent with the SM values.

\section{Flavor changing neutral current}

Top Flavor Changing Neutral Current (FCNC) is the process where a top quark decays to another up-type quark, emitting an electrically neutral particle. This process is allowed at loop-level in the SM though it is highly suppressed by the Glashow-Iliopoulos-Maiani mechanism, leading to branching ratios of $10^{-12}-10^{-15}$ depending on the emitted neutral particle. Many BSM scenarios predict enhanced FCNC with branching ratios up to $\approx 10^{-3}$ bringing it within LHC reach. Two FCNC processes have been studied by CMS with $35.9 \mathrm{fb}^{-1}$ of proton-proton data collected at 

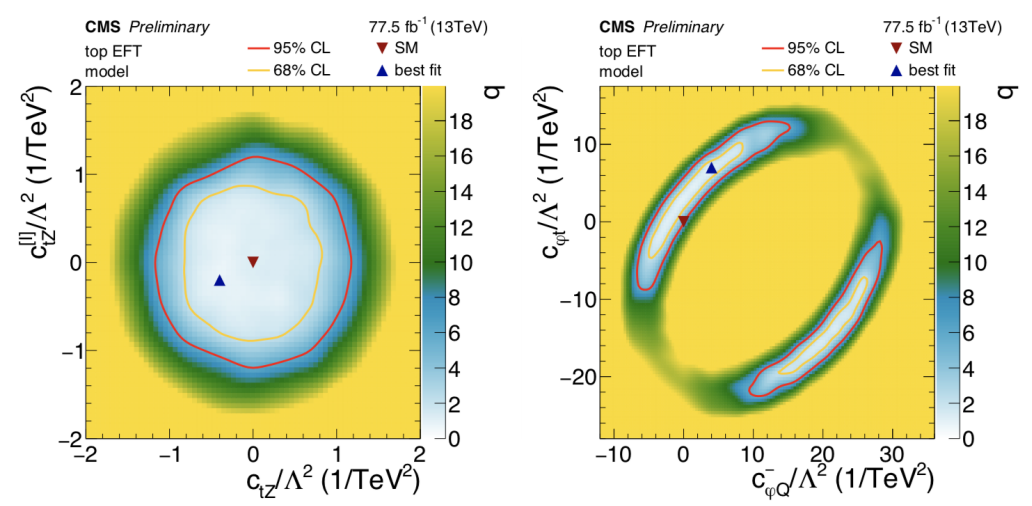

Figure 3: Results of scans in two 2D planes obtain from binned likelihood fit of the yields in each signal/control region described in the text. The color map reflects the negative log-likelihood ratio $q$ w.r.t the best-fit value [9].

$\sqrt{s}=13 \mathrm{TeV}$ : the case where the emitted neutral particle is a Higgs boson (tHq) decaying to two bquarks [10] and the one where it is a Z boson (tZq) decaying to two same flavor leptons (electron or muon) [11]. Both analyses consider the two scenarios where the up-type quark q is a $u$ or a $c$ quark. If one assumes the presence of a new coupling tHq or $\mathrm{tZq}$ allowing for the FCNC decay of the top quark, the associated production of a $\mathrm{Z} / \mathrm{H}$ boson together with a single top quark involving this coupling has also to be considered when deriving the signal contribution. This process known as single-top FCNC leads to final state signature with one less jet compared to the top pair production where one top decay via FCNC ( $t \bar{t}$ FCNC). Both analyses consider single-top FCNC and $t \bar{t}$ FCNC together to derive the signal templates. The tHq analysis requires events with at least one lepton and three jets among which minimum two are b-tagged and performs event reconstruction using both a kinematic fit and a BDT discriminant. Further categorization is applied w.r.t. the number of (b-)jets and other BDT's are trained for signal to background separation. Upper limits on the signal production cross section are derived using the BDT discriminant and re-interpreted in terms of the FCNC branching ratios as shown on the left-hand side plot of Fig. 4.
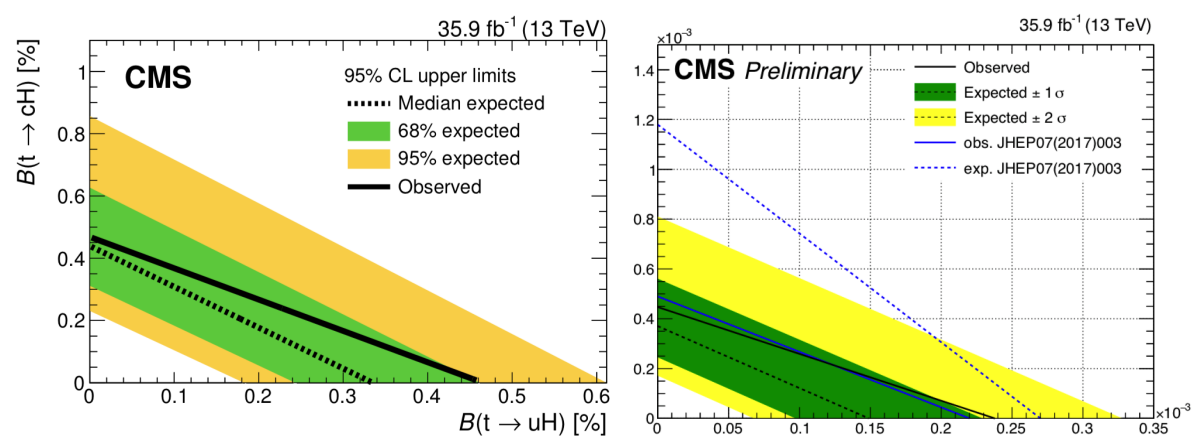

Figure 4: Exclusion regions at 95\% CL on the FCNC branching fraction for $t \rightarrow q H$ (left) [10] and $t \rightarrow q Z$ (right) [11]. In both cases, the $\mathrm{y}$-axis shows $t \rightarrow c X$ while the $\mathrm{x}$-axis shows $t \rightarrow u X$.

The $\mathrm{tZq}$ analysis studies events with three leptons among which one same flavor opposite charge pair is present and with a number of jet between one and three. A categorization is per- 
formed based on the lepton content and signal/control regions are defined based on the jet content. Exclusion limits shown on the right-hand side plot of Fig. 4 are derived from BDT distributions trained to differentiate the FCNC signals from SM backgrounds.

\section{Summary}

Several CMS searches and measurements interpreting results in the EFT framework to constrain operators affecting top quark processes have been presented. The most recent results on FCNC interactions with a top and a Higgs or a $\mathrm{Z}$ boson have also been summarized.

\section{References}

[1] S. Chatrchyan et al. "The CMS experiment at the CERN LHC". JINST, 3:S08004, 2008.

[2] CMS Collaboration. "Search for new physics in top quark production in dilepton final states in proton-proton collisions at $\sqrt{s}=13 \mathrm{TeV}^{\prime}$. Physics Analysis Summary CMS-TOP-17-020-003, 2019. https://cds.cern.ch/record/2669017.

[3] CMS Collaboration. "Measurements of $t \bar{t}$ differential cross sections in proton-proton collisions at $\sqrt{s}$ $=13 \mathrm{TeV}$ using events containing two leptons". Journal of High Energy Physics, 2019(2):149, Feb 2019.

[4] CMS Collaboration. "Measurement of the top quark polarization and t⿱t spin correlations in dilepton final states at $\sqrt{s}=13 \mathrm{TeV}^{\text {“. }}$ Physics Analysis Summary CMS-PAS-TOP-18-006, 2018.

http://cds.cern.ch/record/2649926.

[5] CMS Collaboration. "Search for standard model production of four top quarks with the single-lepton and opposite-sign dilepton final states in proton-proton collisions at $\sqrt{s}=13 \mathrm{TeV}$ “. Physics Analysis Summary CMS-PAS-TOP-17-019, 2019. http://cds. cern. ch/record/2666712.

[6] CMS Collaboration. "Search for standard model production of four top quarks with same-sign and multilepton final states in proton-proton collisions at $\sqrt{s}=13 \mathrm{TeV}$ “. The European Physical Journal C, 78(2):140, Feb 2018.

[7] A. Alloul, N. D. Christensen, C. Degrande, C. Duhr, and B. Fuks. "FeynRules 2.0 - A complete toolbox for tree-level phenomenology“. Computer Physics Communications, 185(8):2250 - 2300, 2014.

[8] J. Alwall, R. Frederix, S. Frixione, V. Hirschi, F. Maltoni, O. Mattelaer, H.-S. Shao, T. Stelzer, P. Torrielli, and M. Zaro. "The automated computation of tree-level and next-to-leading order differential cross sections, and their matching to parton shower simulations“. Journal of High Energy Physics, 2014(7):79, Jul 2014.

[9] CMS Collaboration. "Measurement of top quark pair production in association with a $\mathrm{Z}$ boson in proton-proton collisions at $\sqrt{s}=13 \mathrm{TeV}^{\text {“. }}$ Physics Analysis Summary CMS-PAS-TOP-18-009, 2019. http: //cds. cern.ch/record/2666205.

[10] CMS Collaboration. "Search for the flavor-changing neutral current interactions of the top quark and the Higgs boson which decays into a pair of b quarks at $\sqrt{s}=13 \mathrm{TeV}$ “. Journal of High Energy Physics, 2018(6):102, Jun 2018.

[11] CMS Collaboration. "Search for flavour changing neutral currents in top quark production and decays

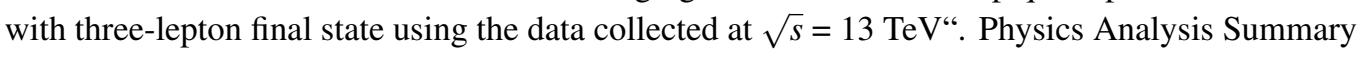
CMS-PAS-TOP-17-017, 2017. https://cds.cern.ch/record/2292045. 